\title{
TRATAMIENTO ELECTROQUÍMICO DE UNA EMULSIÓN ACUOSA DE ÁCIDO PALMÍTICO EMPLEANDO UN ELECTRODO DE FIELTRO GRAFÍTICO MODIFICADO CON HIERRO
}

\author{
Ángel G. Castro ${ }^{\mathrm{a}, \mathrm{b}}$, Maynard J. Kong ${ }^{\mathrm{a}^{*}}$
}

\begin{abstract}
RESUMEN
En el presente estudio proponemos el uso de ánodos de fieltro grafítico recubiertos con hierro para controlar electroquímicamente la cantidad del agente coagulante, en forma del par $\mathrm{Fe}(\mathrm{II}) / \mathrm{Fe}(\mathrm{III})$, que se introduce en la fase acuosa. Al mismo tiempo, este par ayuda a catalizar el proceso electro-Fenton mientras que $\mathrm{H}_{2} \mathrm{O}_{2}$ es generado catódicamente.

Aquí se reportan las condiciones para modificar catódicamente la superficie del fieltro grafítico, así como su caracterización mediante microscopía SEM-EDS y difracción por rayos X (DRX). Luego se emplea este material como ánodo que actúa como fuente del par $\mathrm{Fe}(\mathrm{II}) / \mathrm{Fe}(\mathrm{III})$, en tanto que un fieltro sin modificar actúa como cátodo generador de $\mathrm{H}_{2} \mathrm{O}_{2}$. Bajo estas condiciones, mediante permanganometría, se cuantifica el contenido de $\mathrm{H}_{2} \mathrm{O}_{2}$ producido en función del tiempo. Por último, el fieltro grafítico fue aplicado en el tratamiento electroquímico de emulsiones sintéticas de ácido palmítico estabilizadas en medio acuoso, aplicando densidades de corriente en el rango de $5-20 \mathrm{~mA} / \mathrm{cm}^{2}$, logrando reducir significativamente los niveles de demanda química de oxígeno (DQO) y de turbidez nefelométrica (NTU) en casi $75 \%$ y $90 \%$, respectivamente, en unos 30 minutos.
\end{abstract}

Palabras clave: fieltro de grafito, modificación de electrodos, ácido palmítico.

\section{ELECTROCHEMICAL TREATMENT OF AN AQUEOUS PALIMITIC ACID EMULSION BY MEANS OF AN IRON MODIFIED GRAPHITE FELT ELECTRODE}

\begin{abstract}
In the present study we propose the use of graphitic felt anodes coated with iron to control electrochemically the amount of coagulating agent, in the form of the $\mathrm{Fe}(\mathrm{II}) / \mathrm{Fe}$ (III) pair, which is introduced into the aqueous phase. At the same time, this pair helps catalyze the electro-Fenton process while $\mathrm{H}_{2} \mathrm{O}_{2}$ is generated cathodically.

a Departamento de Ciencias - Sección Química, Pontificia Universidad Católica del Perú (PUCP). Av. Universitaria 1801, Lima 32. Lima-Perú.

b Área de Ciencias, Universidad Peruana de Ciencias Aplicadas (UPC). Av. La Marina 2810, Lima 32.

Lima-Perú.

*mjkong@pucp.edu.pe.
\end{abstract}


Here are reported the conditions to modify cathodically the graphite felt surface, as well as its characterization by SEM-EDS microscopy and X-ray diffraction (XRD). This material is then used as an anode that acts as a source for the $\mathrm{Fe}(\mathrm{II}) / \mathrm{Fe}(\mathrm{III})$ pair, while an unmodified felt acts as a $\mathrm{H}_{2} \mathrm{O}_{2}$ generator. Under these conditions, by means of permanganometry, the $\mathrm{H}_{2} \mathrm{O}_{2}$ content produced in function of time is quantified. Finally, the graphite felt was applied in the electrochemical treatment of synthetic emulsions of palmitic acid stabilized in aqueous medium, applying current densities in the range of $5-20 \mathrm{~mA} / \mathrm{cm}^{2}$, achieving a significant reduction in the levels of chemical oxygen demand (COD) ) and nephelometric turbidity (NTU) by almost $75 \%$ and $90 \%$, respectively, in about 30 minutes.

Key words: graphite felt, electrode modification, palmitic acid.

\section{INTRODUCCIÓN}

Los procesos avanzados de oxidación (PAO) ofrecen métodos muy eficaces para degradar contaminantes en medios acuosos ${ }^{1-7}$. Entre ellos, se destaca el método electro-Fenton, donde el peróxido de hidrógeno se genera catódicamente y luego se convierte catalíticamente por $\mathrm{Fe}(\mathrm{II})$ en radicales con alto poder oxidante $\mathrm{e}^{2,5-7}$. Varios estudios han demostrado la gran capacidad de estos radicales para degradar compuestos orgánicos como productos farmacéuticos, colorantes, pesticidas, compuestos aromáticos, fenoles, ácidos carboxílicos, etc., que son altamente resistentes al tratamiento con métodos convencionales ${ }^{2,5-7}$. Entre los contaminantes presentes en las aguas residuales, el tratamiento de ácidos grasos provenientes de diferentes tipos de industria, como alimentos, acuicultura, pesca, etc., representan un serio problema ambiental ${ }^{8-10}$. En la presente investigación se propone estudiar la preparación y aplicación de electrodos de fieltro grafítico, modificados por deposición de hierro, para el tratamiento en medio acuoso de ácido palmítico ${ }^{11}$. Esta especie servirá como una molécula de prueba para modelar el comportamiento de los ácidos grasos. El hierro presente en el fieltro influye tanto en la oxidación como en la coagulación del ácido palmítico disperso en la fase acuosa $^{11}$.

\section{PARTE EXPERIMENTAL}

\section{Modificación y caracterización del fieltro grafítico}

En este estudio se empleó fieltro grafitico a base de rayón (Carbopon-B22 de Sohim Innovativ Materials) de $3 \mathrm{~mm}$ de espesor. Mediante la deposición catódica de películas metálicas de hierro sobre fieltro grafítico se procedió a modificar $4 \mathrm{~cm}^{2}$ de este substrato, aplicando una densidad de corriente de $90 \mathrm{~mA} / \mathrm{cm}^{2}$ (fuente digital de voltaje Ledblink HY3003) durante 20 minutos, en contacto con una solución electrolítica de Fe $\left(\mathrm{NH}_{4}\right)_{2}\left(\mathrm{SO}_{4}\right)_{2}$ ( $\mathrm{sal}$ de Mohr $250 \mathrm{~g} / \mathrm{L}$, $\mathrm{CDH} 99 \%$ ) en medio acuoso y $\mathrm{pH}$ ajustado a 3 con $\mathrm{H}_{2} \mathrm{SO}_{4}$. En esta celda de electrodeposición se completó el circuito electroquímico con un ánodo de acero inoxidable C-304L de $2 \mathrm{~mm}$ de espesor. 
De ahí se probó la estabilidad del fieltro grafítico recién modificado catódicamente, al someterlo a ensayos de oxidación anódica durante 30 minutos (fuente Ledblink HY3003). Se determinó el contenido de Fe disuelto en la solución electrolítica al analizar sus alícuotas mediante absorción atómica a la llama (Perkin Elmer Pinaacle 900H). Este contenido fue identificado como una fracción o porcentaje respecto al total de Fe que recubre el fieltro modificado, y cuya cantidad fue determinada al someter todo el fieltro modificado a una digestión ácida prolongada. Aquí también se determinó el contenido de Fe oxidado y disuelto mediante espectroscopia de absorción atómica a la llama. Ambos resultados del contenido de hierro del recubrimiento sobre el fieltro, el total por digestión ácida y la cantidad parcial por oxidación anódica, son reportados en la tabla 1.

El electrodo modificado fue retirado de la celda de electrodeposición y, luego de unas semanas, fue caracterizado ex situ mediante microscopía SEM-EDS (FEI Quanta 650) así como difracción por rayos X (DRX, Bruker ECO ENDEAVOR). Los resultados de estas caracterizaciones se muestran en las figuras 3,4 y 5 , y en las tablas 2 y 3 .

\section{Ensayos de generación electrolítica de $\mathrm{H}_{2} \mathrm{O}_{2}$}

Se estudió en función del tiempo la capacidad del fieltro grafítico recién recubierto con hierro en contribuir como contra-electrodo (ánodo) en la generación electrolítica de $\mathrm{H}_{2} \mathrm{O}_{2}$. En esta celda (figura 1), se tiene como electrodo de trabajo responsable directo de la generación catódica de $\mathrm{H}_{2} \mathrm{O}_{2}$ al fieltro grafítico no modificado en contacto con $\mathrm{Na}_{2} \mathrm{SO}_{4}$ acuoso $(0,10$ mol/L, Merck 99,90 \%) acidificado a $\mathrm{pH} 3$ con $\mathrm{H}_{2} \mathrm{SO}_{4}$, y donde se hizo burbujear permanente oxígeno medicinal (AGA 99,5 \%) a flujo constante de 1/16 L/min.

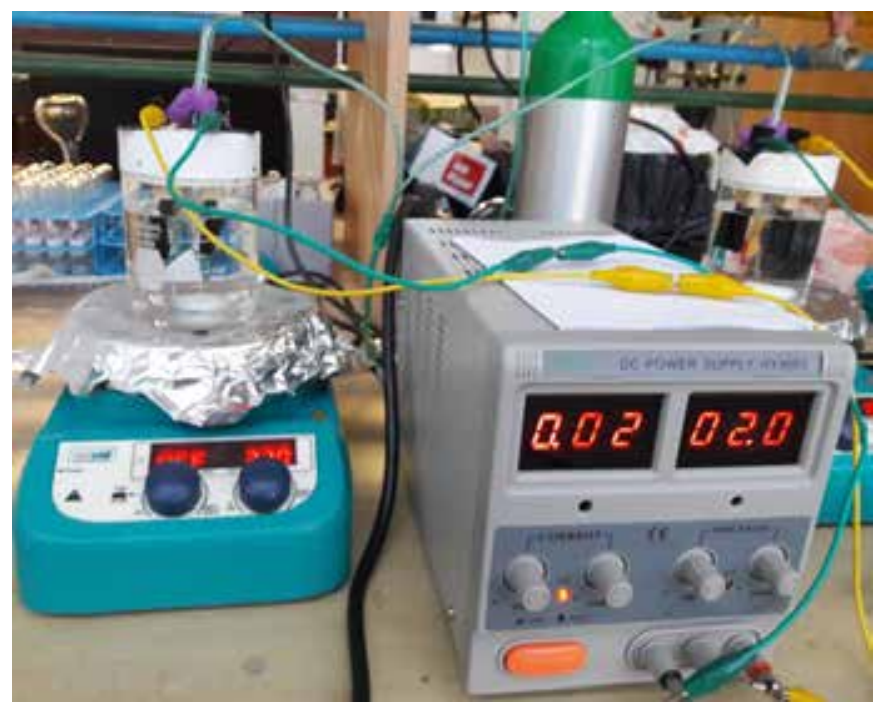

Figura 1. Celda de generación de peróxido de hidrógeno en funcionamiento. 
La generación de $\mathrm{H}_{2} \mathrm{O}_{2}$ se realizó aplicando diferentes densidades de corriente: 5, 10, 15 y 20 $\mathrm{mA} / \mathrm{cm}^{2}$ (fuente Ledblink HY3003). El contenido de $\mathrm{H}_{2} \mathrm{O}_{2}$ generado en la celda se determinó mediante permanganimetría. Se tomó una alícuota de $5 \mathrm{~mL}$ de la celda electrolítica donde se generó $\mathrm{H}_{2} \mathrm{O}_{2}$ (figura 1), en intervalos de 30 minutos hasta completar 180 minutos, y se procedió a titular con una solución $0,02 \mathrm{~mol} / \mathrm{L}$ de $\mathrm{KMnO}_{4}$ (Merck, 99,80 \%). El perfil de evolución de $\mathrm{H}_{2} \mathrm{O}_{2}$ en el tiempo está representado en la figura 7.

\section{Tratamiento electroquímico de la emulsión acuosa de ácido palmítico}

Como modelo en la aplicación del fieltro modificado en el tratamiento electrolítico de aceites y grasas en efluentes acuosos, se preparó una mezcla acuosa que contenía simultáneamente ácido palmítico $(0,10 \mathrm{~g} / \mathrm{L}$, Merck $65 \%)$ con $\mathrm{Na}_{2} \mathrm{SO}_{4}(0,10 \mathrm{~mol} / \mathrm{L}$, Merck 99,90 \%) y Tritón X-100 $(0,15915 \mathrm{~g} / \mathrm{L}$, Merck $99 \%)$ como estabilizante de la emulsión. La mezcla fue primero agitada magnéticamente a $1400 \mathrm{rpm}$ en una cocinilla (ISOLAB Laborgerate $\mathrm{GmbH}$ ) a $80 \mathrm{oC}$ hasta lograr la fusión del ácido palmítico, y luego se procedió a agitar la mezcla resultante en un equipo de ultrasonido (MRC Scientific Instruments) por una hora más a temperatura ambiente. Con ello se obtuvo una emulsión turbia y estable de ácido palmítico en agua (figura 2).

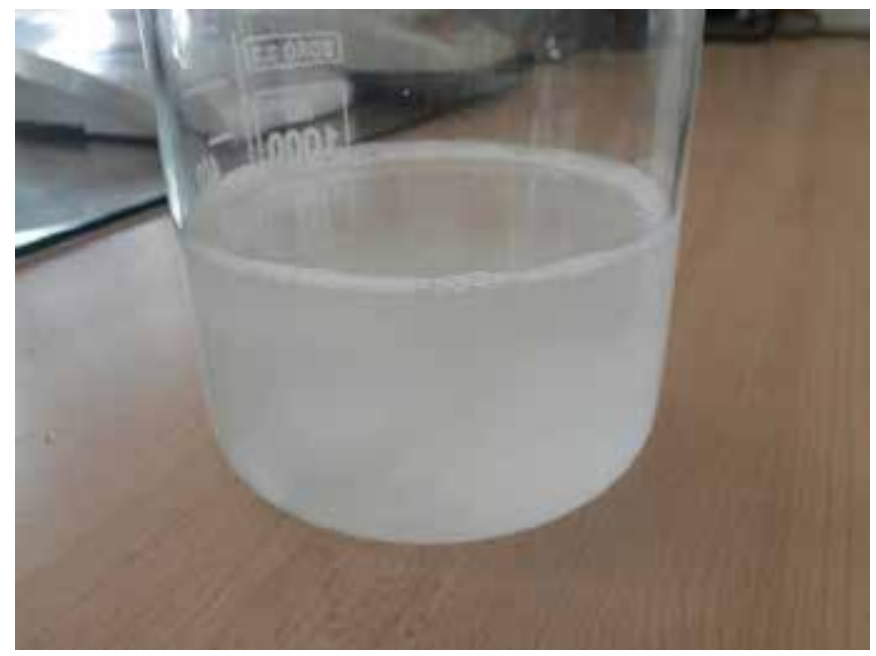

Figura 2. Muestra de ácido palmítico en forma de emulsión acuosa.

Tales muestras sintéticas fueron luego tratadas electroquímicamente en una celda similar a la usada para la generación de $\mathrm{H}_{2} \mathrm{O}_{2}$, aplicando densidades de corriente de $5,10,15$ y $20 \mathrm{~mA} / \mathrm{cm}^{2}$ (fuente Ledblink HY3003) entre un cátodo del fieltro grafítico no modificado y un ánodo del fieltro recubierto con hierro. Durante los tratamientos electroquímicos de la mezcla, se procedió a retirar alícuotas cada 10 minutos hasta completar 60 minutos de ensayo para ir cuantificando la turbidez nefelométrica (turbidímetro Lovibond Turbicheck) y demanda química de oxígeno (DQO) correspondientes a cada uno de estos tiempos. Estos resultados están registrados en las tablas 5 y 6, así como en las figuras 9 y 10. En la figura 8, se muestra el aspecto de algunas de estas muestras para tratamientos electroquímicos de 10 a 60 minutos para una densidad de corriente de $10 \mathrm{~mA} / \mathrm{cm}^{2}$. 
Para la determinación colorimétrica de la DQO en las muestras tratadas electroquímicamente, se tomaron alícuotas de 2,5 mL de la fase superior del contenido almacenado en los viales que contenían las muestras extraídas de la celda electrolítica, y se vertieron en tubos de ensayo con un exceso conocido de solución estándar de K2Cr2O7 (JT Baker 99,00 \%) en presencia de ácido sulfúrico concentrado. Luego se colocaron en un digestor (NANOCOLOR VARIO C2) por 2 horas a $150{ }^{\circ} \mathrm{C}$.

Puesto que la introducción de Tritón X-100 altera el valor total de DQO en la mezcla acuosa, ya que incluiría la cuantificación tanto del ácido palmítico como del surfactante, se tuvo que corregir esta determinación de DQO respecto a una mezcla en blanco de la solución acuosa de $\mathrm{Na}_{2} \mathrm{SO}_{4}$ con el surfactante, pero sin incluir ácido palmítico. Se prepararon varias muestras estándares de ácido palmítico bajo estas condiciones y, en todos los casos, se determinó el valor de DQO fotométricamente (espectrofotómetro UV/Vis Agilent 8453) midiendo la absorbancia visible a $610 \mathrm{~nm}$, y restando la absorbancia correspondiente al blanco del surfactante sin el ácido palmítico.

En la tabla 6 y figura 10 se reportan los valores de DQO correspondientes a las muestras de ácido palmítico tratadas electroquímicamente a diferentes densidades de corriente.

\section{RESULTADOS Y DISCUSIÓN}

\section{Modificación y caracterización del fieltro grafítico}

En la micrografía SEM de la figura 3 se aprecia que, alrededor de la ramificación de fibras propias del fieltro grafítico, se han depositado láminas grises de hierro metálico que progresivamente se han ido oxidado al aire.

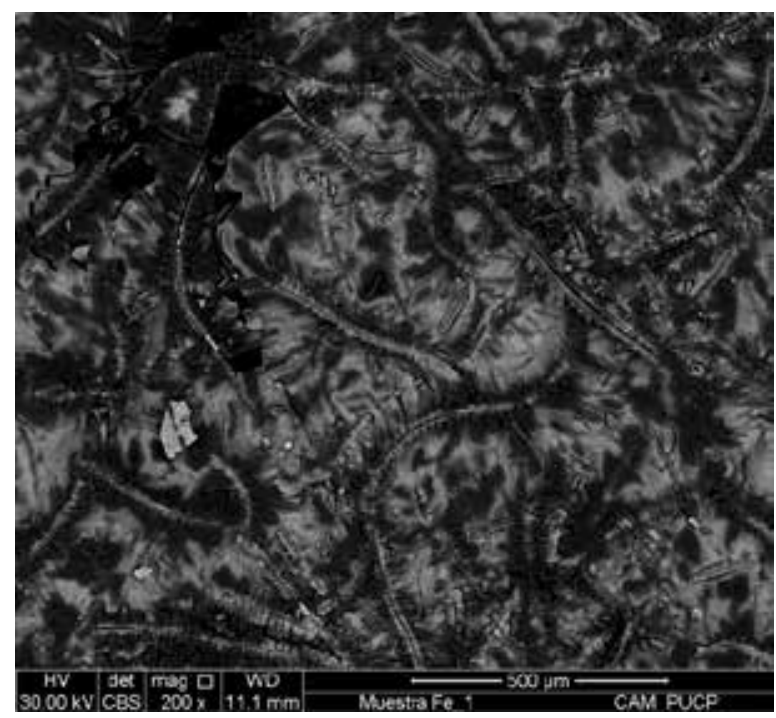

Figura 3. Micrografía SEM del recubrimiento del fieltro grafítico. 
A continuación (tabla 1) se reporta el contenido de Fe determinado por absorción atómica a la llama, tanto del recubrimiento sometido a digestión ácida prolongada como del recubrimiento tratado mediante oxidación anódica.

Tabla 1. Resultados por absorción atómica para el hierro depositado sobre el fieltro de grafito y para el hierro liberado en la solución utilizando como ánodo el fieltro modificado.

\begin{tabular}{|c|c|c|c|c|c|}
\hline Muestra & Absorbancia & $\begin{array}{l}\text { Concentración } \\
\text { Fe (mg/L) para } \\
\text { la muestra } \\
\text { diluida }\end{array}$ & Dilución & $\begin{array}{l}\text { Concentración } \\
\text { Fe }(\mathrm{mg} / \mathrm{L}) \\
\text { para la muestra } \\
\text { sin diluir }\end{array}$ & $\begin{array}{c}\text { Masa } \\
\text { del Fe } \\
(\mathrm{g})\end{array}$ \\
\hline $\begin{array}{l}\text { Digestión } \\
\text { ácida } \\
\text { del } \\
\text { fieltro } \\
\end{array}$ & 0,1677 & 1,927 & $200 \mu \mathrm{L} / 100 \mathrm{~mL}$ & 963,504 & 0,096 \\
\hline $\begin{array}{c}\text { Fe } \\
\text { liberado } \\
\text { en la } \\
\text { solución } \\
\text { por el } \\
\text { ánodo } \\
\end{array}$ & 0,0314 & 0,269 & $80 \mu \mathrm{L} / 100 \mathrm{~mL}$ & 336,071 & 0,034 \\
\hline
\end{tabular}

Asumiendo que mediante la digestión ácida se remueve en su totalidad el recubrimiento de hierro (0,096 g), el porcentaje de hierro que queda sobre el fieltro luego de su remoción anódica al medio electrolítico (0,034 g) durante 30 minutos es de 64,58\%. Este es el porcentaje del recubrimiento de hierro sobre el fieltro que resiste a la oxidación anódica. El 35,41\% restante de hierro corresponde a la cantidad del metal que se oxida anódicamente y pasa a la solución electrolítica. Asumiendo que el hierro electrodepositado en este recubrimiento sigue la estructura correspondiente a la ferrita $(\alpha-\mathrm{Fe})$, se estima que bajo estas condiciones se logra electro-depositar láminas metálicas en una película con espesor del orden de $40 \mu \mathrm{m}$.

El espectro EDS (figura 4) revela los elementos presentes en el recubrimiento formado al modificar catódicamente la superficie del fieltro grafítico.

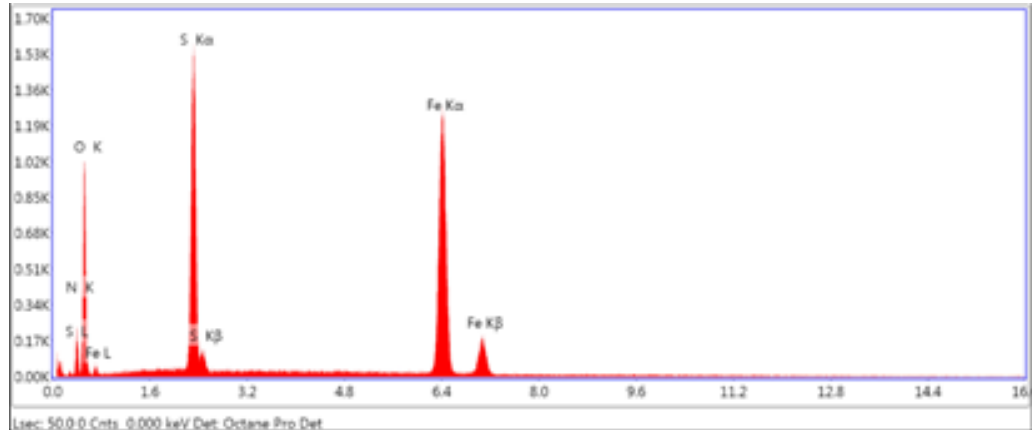

Figura 4. Espectro EDS para el recubrimiento depositado catódicamente sobre el fieltro grafítico. 
En el espectro EDS (figura 4) para el recubrimiento depositado catódicamente se identifica la presencia de $\mathrm{Fe}, \mathrm{S}, \mathrm{O}$ y $\mathrm{N}$. Las señales de $\mathrm{S}, \mathrm{N}$ provendrían de la sal de Mohr utilizada como precursor para la electro-deposición del Fe depositado. La composición elemental determinada por EDS no permite identificar directamente los compuestos químicos que conforman el recubrimiento de hierro depositado catódicamente sobre el fieltro grafítico, por lo que se recurrió a los análisis por difracción de rayos X (DRX). De los análisis por EDS se logró determinar la composición elemental del recubrimiento sobre el fieltro grafítico (tabla 2).

Tabla 2. Elementos determinados por EDS en el recubrimiento del fieltro grafítico recubierto catódicamente.

\begin{tabular}{cccc}
\hline Elemento & \% en masa & \% atómico & \% de error (masa) \\
\hline $\mathrm{N}$ & 16,69 & 25,29 & 10,73 \\
$\mathrm{O}$ & 41,74 & 55,39 & 9,89 \\
$\mathrm{~S}$ & 12,49 & 8,27 & 4,27 \\
$\mathrm{Fe}$ & 29,09 & 11,06 & 1,64 \\
\hline
\end{tabular}

En la figura 5 se observa el difractograma correspondiente a una muestra en polvo del recubrimiento raspado del fieltro modificado por deposición catódica. Se aprecia que el recubrimiento contiene varias fases sólidas, principalmente a base de hierro, tales como

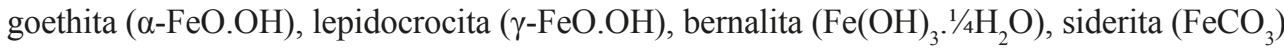
y quenstedtita $\left(\mathrm{Fe}_{2}\left(\mathrm{SO}_{4}\right)_{3} \cdot 11\left(\mathrm{H}_{2} \mathrm{O}\right)\right)$, siendo la fase de goethita la predominante con casi $40 \%$ de la composición en masa (tabla 3 y figura 6), seguida por casi $30 \%$ de fases amorfas. Se observa también un alto contenido de nitrógeno debido a cationes de amonio, provenientes de la sal de Mohr empleada como precursor, atraídos hacia el fieltro durante la deposición catódica, estabilizados en las fases sólidas formadas, y que no pudieron ser retirados en la etapa de enjuague de este recubrimiento. 


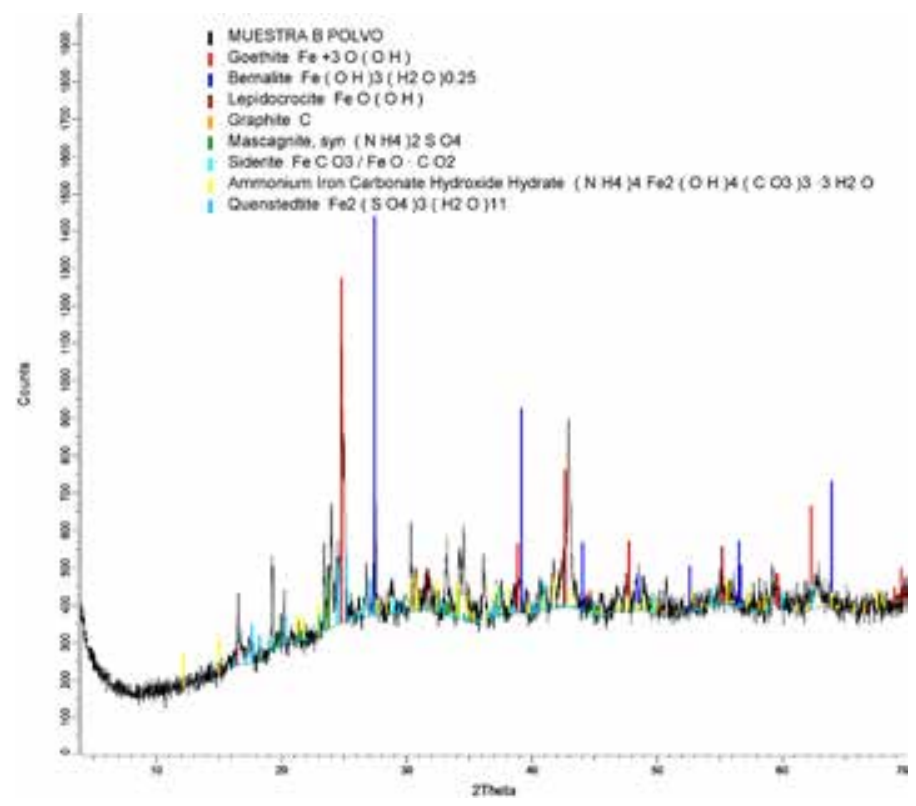

Figura 5. Difractograma en la identificacion de los minerales presentes en el recubrimiento catódico raspado del fieltro grafítico.

Tabla 3. Composición porcentual del recubrimiento del fieltro grafítico.

\begin{tabular}{|c|c|c|}
\hline & Fases identificadas & $\%$ en masa \\
\hline \multirow{9}{*}{$\begin{array}{c}\text { Distribución } \\
\text { de fases } \\
\text { sólidas } \\
\text { cristalinas }\end{array}$} & Goethita, $\alpha-\mathrm{FeO} . \mathrm{OH}$ & 40,15 \\
\hline & Mascagnita, $\left(\mathrm{NH}_{4}\right)_{2} \mathrm{SO}_{4}$ & 14,96 \\
\hline & Lepidocrocita, $\gamma$-FeO.OH & 6,90 \\
\hline & Quenstedtita, $\mathrm{Fe}_{2}\left(\mathrm{SO}_{4}\right)_{3} .11\left(\mathrm{H}_{2} \mathrm{O}\right)$ & 3,96 \\
\hline & Siderita, $\mathrm{FeCO}_{3}$ & 1,61 \\
\hline & Carbono grafítico & 1,06 \\
\hline & Bernalita, $\mathrm{Fe}(\mathrm{OH})_{3} .{ }^{1} / 4 \mathrm{H}_{2} \mathrm{O}$ & 0,87 \\
\hline & $\begin{array}{l}\text { Hidróxido-carbonato amoniacal de } \mathrm{Fe}(\mathrm{III}) \text {, } \\
\left(\mathrm{NH}_{4}\right)_{4} \mathrm{Fe}_{2}(\mathrm{OH})_{4}\left(\mathrm{CO}_{3}\right)_{3} .3\left(\mathrm{H}_{2} \mathrm{O}\right)\end{array}$ & 0,73 \\
\hline & Distribución de fases amorfas & 29,78 \\
\hline & Total & 100,00 \\
\hline
\end{tabular}




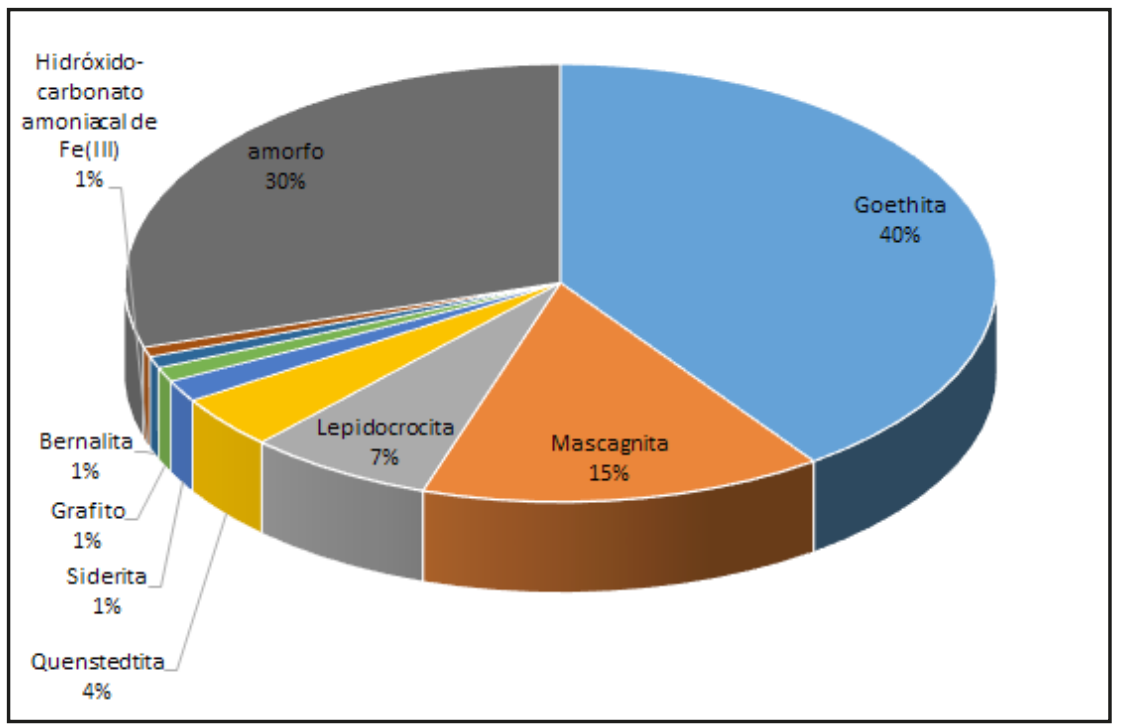

Figura 6. Representación gráfica de la composición porcentual del recubrimiento catódico de hierro sobre el fieltro grafítico.

\section{Ensayos de generación electrolítica de $\mathrm{H}_{2} \mathrm{O}_{2}$.}

De ahí, se probó la estabilidad del fieltro grafítico modificado catódicamente, sometiendo este electrodo a ensayos de oxidación anódica durante 30 minutos. El hierro recubierto resiste su disolución en $64,6 \%$. El restante $35,4 \%$ de hierro oxidado y disuelto al medio electrolítico mostraría luego tener la capacidad de actuar como coagulante y, a su vez, como catalizador de la reacción electro-Fenton.

Se estudió la capacidad del fieltro grafítico recién recubierto con hierro en generar $\mathrm{H}_{2} \mathrm{O}_{2}$ catódicamente en función del tiempo. Estos ensayos se llevaron a cabo en otra celda, provista de un ánodo del fieltro grafítico en contacto con $\mathrm{Na}_{2} \mathrm{SO}_{4}$ acuoso acidificado a $\mathrm{pH}$ 3. Las pruebas se realizaron a diferentes densidades de corriente $\left(5,10,15\right.$, y $\left.20 \mathrm{~mA} / \mathrm{cm}^{2}\right)$. Bajo estas condiciones, el contenido de $\mathrm{H}_{2} \mathrm{O}_{2}$ logró aumentar inicialmente con la densidad de corriente aplicada, pero tiende a decaer luego de 120 minutos de aplicación (tabla 4 y figura 7). 
Tabla 4. Resultados de la generación de peróxido de hidrógeno a diferentes densidades de corriente eléctrica $\left(\mathrm{mA} / \mathrm{cm}^{2}\right)$ utilizando como ánodo fieltro de grafito recubierto catódicamente con hierro.

\begin{tabular}{ccccc}
\hline \multicolumn{5}{c}{$\left[\mathrm{H}_{2} \mathrm{O}_{2}\right] \mathrm{mM}$} \\
$\mathrm{t}(\mathrm{min})$ & $5 \mathrm{~mA} / \mathrm{cm}^{2}$ & $10 \mathrm{~mA} / \mathrm{cm}^{2}$ & $15 \mathrm{~mA} / \mathrm{cm}^{2}$ & $20 \mathrm{~mA} / \mathrm{cm}^{2}$ \\
\hline 30 & 0,106 & 0,212 & 0,318 & 0,424 \\
60 & 0,106 & 0,212 & 0,318 & 0,424 \\
90 & 0,159 & 0,318 & 0,424 & 0,53 \\
120 & 0,159 & 0,424 & 0,53 & 0,742 \\
150 & 0,109 & 0,424 & 0,318 & 0,636 \\
180 & 0,109 & 0,318 & 0,212 & 0,424 \\
\hline
\end{tabular}

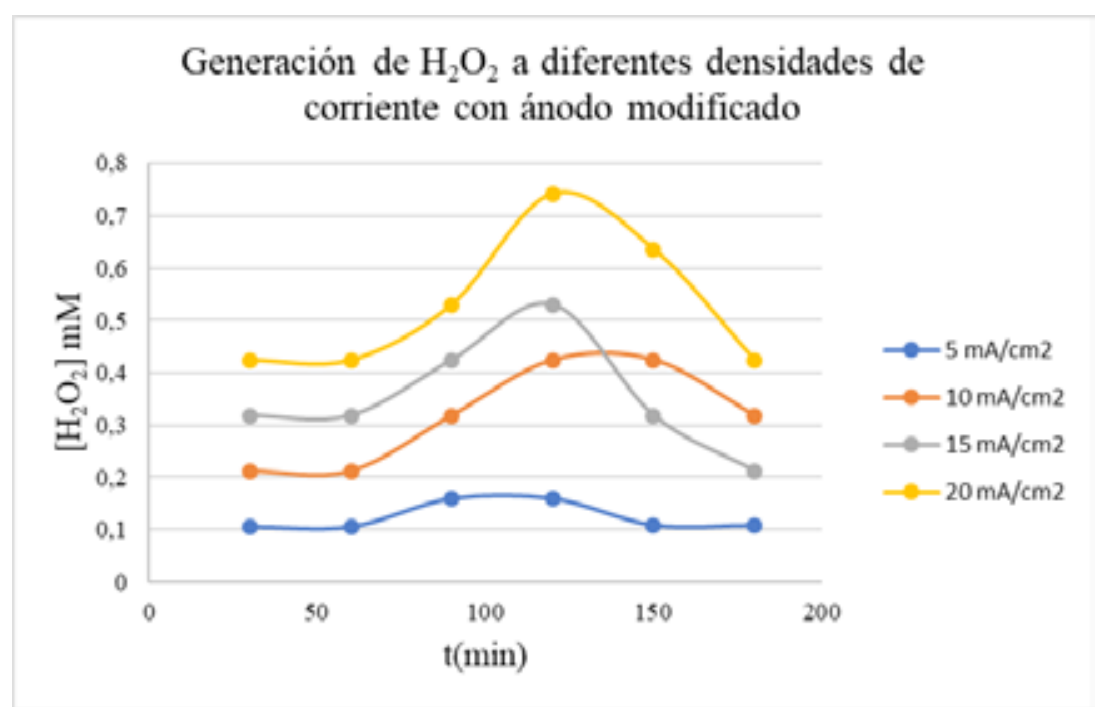

Figura 7. Comparación de la generación de peróxido de hidrógeno a diferentes densidades de corriente utilizando cátodo de fieltro de grafito sin recubrir y ánodo de fieltro de grafito recubierto catódicamente con hierro. 


\section{Tratamiento electroquímico de la emulsión acuosa de ácido palmítico}

Como modelo en la aplicación del fieltro modificado en el tratamiento electrolítico de aceites y grasas en efluentes acuosos, se preparó una mezcla acuosa de ácido palmítico en forma de una emulsión estabilizada con Tritón X-100. Esta muestra sintética fue tratada electrolíticamente en una celda similar a la usada para la generación de $\mathrm{H}_{2} \mathrm{O}_{2}$. Mediante este tratamiento electrolítico se redujo significativamente los niveles de demanda química de oxígeno (DQO) y de turbidez nefelométrica (NTU) en tiempos relativamente cortos: casi $75 \%$ de reducción de DQO y $90 \%$ de reducción en unos 30 minutos. Estos resultados se justifican por una acción combinada del proceso electro-Fenton iniciado en el cátodo de fieltro grafítico, y de la electro-coagulación inducida por la oxidación y disolución de hierro desde el fieltro grafítico modificado con este metal.

En la figura 8, se muestra el aspecto de algunas de estas muestras para tratamientos electroquímicos de 10 a 60 minutos para una densidad de corriente aplicada de $10 \mathrm{~mA} /$ $\mathrm{cm} 2$. Como se puede observar, para los tiempos menores de tratamiento electroquímico (a la izquierda de figura 8) las mezclas son más turbias y no se aprecian precipitados. Conforme aumenta el tiempo de tratamiento (a la derecha de figura 8) por acción de iones ferrosos provenientes del fieltro modificado, la fase líquida se va aclarando y se forman precipitados marrones al fondo de los viales.

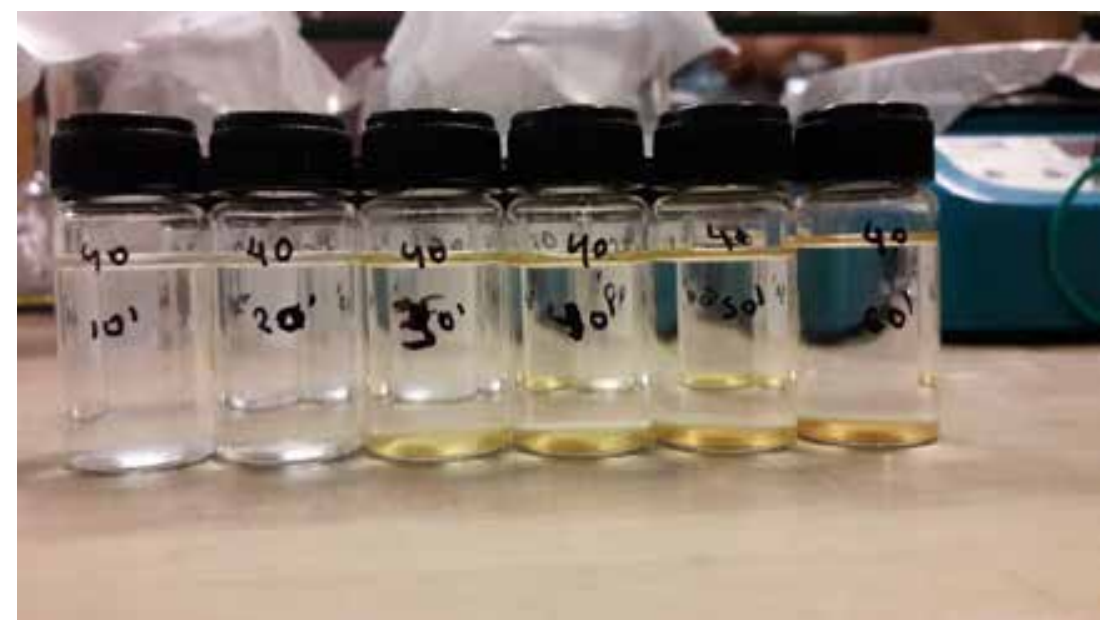

Figura 8. Viales con el producto del tratamiento electroquímico de la emulsión de ácido palmítico estabilizado con Tritón X-100.

La apreciación visual presentada en la figura 8 se confirma de modo cuantitativo al analizar los resultados de turbidez registrados en la tabla 5 y figura 9. Estos resultados indican que, para las cuatro densidades de corriente estudiadas, la turbidez decae rápidamente con el tiempo. Este decaimiento es significativo a partir de los 20 minutos de tratamiento electroquímico, y varía muy poco desde los 30 minutos de aplicación. Con el tratamiento de $15 \mathrm{~mA} / \mathrm{cm}^{2}$ el decaimiento de la turbidez fue mucho más rápido y eficiente que en los demás casos estudiados. 
Tabla 5. Turbidez de la emulsión acuosa del ácido palmítico con Tritón X-100, luego de su tratamiento electroquímico, en función del tiempo y de las densidades de corriente.

\begin{tabular}{ccccc}
\hline \multicolumn{5}{c}{ Turbidez (NTU) } \\
\hline $\mathrm{t}(\mathrm{min})$ & $5 \mathrm{~mA} / \mathrm{cm}^{2}$ & $10 \mathrm{~mA} / \mathrm{cm}^{2}$ & $15 \mathrm{~mA} / \mathrm{cm}^{2}$ & $20 \mathrm{~mA} / \mathrm{cm}^{2}$ \\
\hline 0 & 88 & 88 & 88 & 88 \\
10 & 77,6 & 43 & 8,05 & 19,9 \\
20 & 44,8 & 11,5 & 4,35 & 11,9 \\
30 & 18,7 & 6,55 & 3,32 & 5,67 \\
40 & 18 & 4,69 & 3,3 & 4,93 \\
50 & 17,4 & 3,33 & 2,01 & 4,21 \\
60 & 13,5 & 2,62 & 1,93 & 4,13 \\
\hline
\end{tabular}

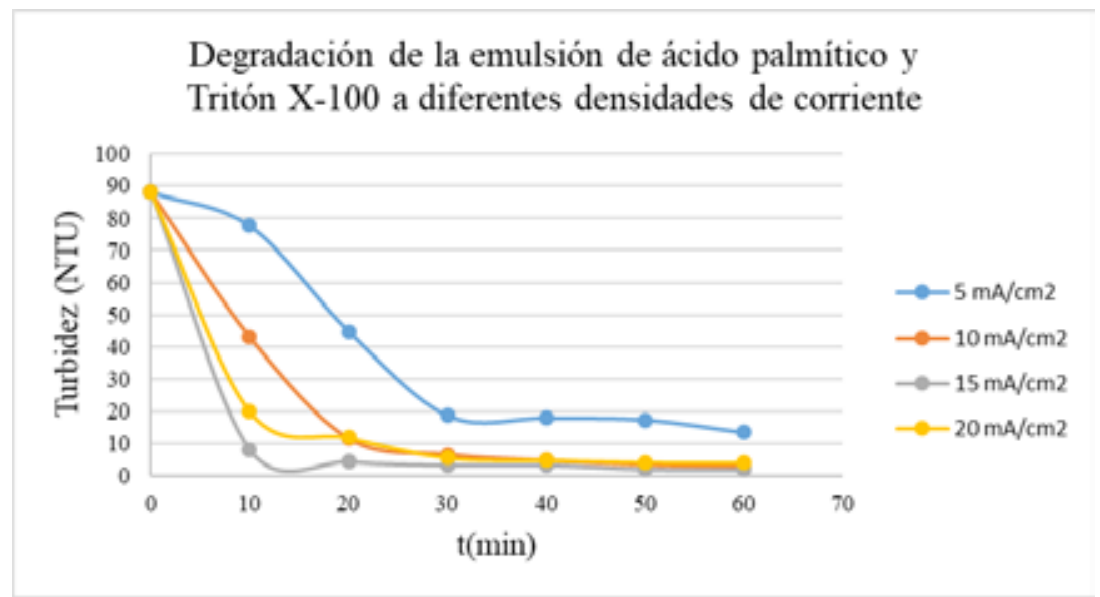

Figura 9. Comparación de la turbidez en la emulsión acuosa de ácido palmítico tratado electroquímicamente a diferentes densidades de corriente.

En la tabla 6 y figura 10 se reporta la evolución de la DQO en función del tiempo para diferentes densidades de corriente aplicadas. Si bien los valores de DQO disminuyen con el tiempo, al igual que en los estudios de variación de turbidez, el patrón y orden de decaimiento en DQO es totalmente distinto a los casos estudiados para la disminución de turbidez, siendo la densidad de corriente de $10 \mathrm{~mA} / \mathrm{cm}^{2}$ el más adecuado para reducir la DQO, y el de $20 \mathrm{~mA} / \mathrm{cm}^{2}$ es el menos indicado para ello. 
Tabla 6. Comparación entre valores de DQO en emulsiones acuosas de ácido palmítico sujetos al tratamiento electroquímico para diferentes tiempos y densidades de corriente.

DQO (mg $\left.\mathrm{O}_{2} / \mathrm{L}\right)$ (emulsión de ácido palmítico con Tritón X-100)

\begin{tabular}{ccccc}
\hline $\mathrm{t}(\mathrm{min})$ & $5 \mathrm{~mA} / \mathrm{cm}^{2}$ & $10 \mathrm{~mA} / \mathrm{cm}^{2}$ & $15 \mathrm{~mA} / \mathrm{cm}^{2}$ & $20 \mathrm{~mA} / \mathrm{cm}^{2}$ \\
\hline 0 & 724,17 & 717,27 & 725,83 & 731,83 \\
10 & 370,30 & 288,23 & 185,70 & 234,40 \\
20 & 206,20 & 113,87 & 170,30 & 203,63 \\
30 & 139,07 & 95,93 & 155,23 & 188,23 \\
40 & 119,03 & 85,70 & 144,10 & 170,30 \\
50 & 113,90 & 67,73 & 136,93 & 162,60 \\
60 & 106,20 & 62,60 & 131,83 & 157,47 \\
\hline
\end{tabular}

Degradación de la emulsión de ácido palmítico y Triton X-100 a diferentes densidades de corriente

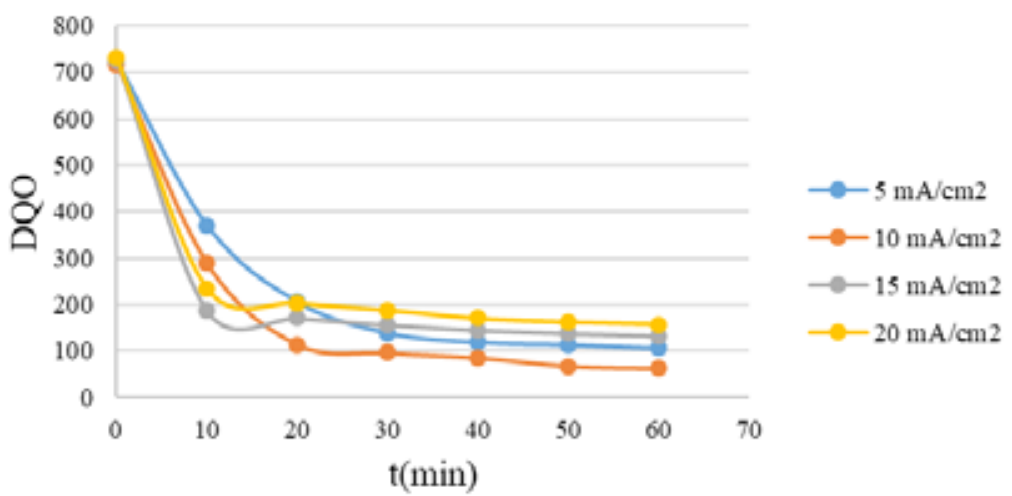

Figura 10. Comparación en la evolución de valores de DQO al tratar electrolíticamente las emulsiones de ácido palmítico con diferentes densidades de corriente. 
Los perfiles de evolución o decaimiento en el tiempo de los parámetros de calidad del efluente, turbidez y DQO, contienen mucha información acerca de los mecanismos que participan en los complejos procesos de tratamiento electroquímico. Una propuesta posible que justifique por qué difieren los patrones de evolución de DQO y turbidez dentro de una misma celda es el hecho de tener dos procesos paralelos que se combinan y compiten entre sí en el tratamiento electroquímico estudiado: a) la generación catódica de $\mathrm{H}_{2} \mathrm{O}_{2}$ que debería ser mucho más activa en reducir los valores de DQO en el tiempo, y b) la oxidación y disolución de hierro presente en el recubrimiento del ánodo del fieltro, que favorecerían más bien disminución activa de la turbidez.

Los niveles de reducción de DQO y turbidez son significativos, alcanzando una disminución de casi $75 \%$ en la demanda química de oxígeno (DQO) y de cerca de $90 \%$ en la turbidez nefelométrica (NTU) en unos 30 minutos.

\section{CONCLUSIONES}

Se determinaron las condiciones experimentales adecuadas para llegar a recubrir el fieltro grafítico mediante deposición catódica de películas metálicas de hierro sobre su superficie, empleando para ello una solución electrolítica de $\mathrm{Fe}\left(\mathrm{NH}_{4}\right)_{2}\left(\mathrm{SO}_{4}\right)_{2}$ a $\mathrm{pH}$ cercano a 3. Se estima que la película de hierro metálico depositado sobre el fieltro tendría un espesor del orden de 40 $\mu \mathrm{m}$ y puede resistir la oxidación anódica durante 30 minutos en 64,6\%. El restante 35,4 \% de hierro oxidado y disuelto al medio electrolítico mostraría luego tener la capacidad de actuar como coagulante y, a su vez, como catalizador de la reacción electro-Fenton.

Al retirar los electrodos modificados de la celda de electrodeposición, las caracterizaciones ex situ (SEM-EDS, DRX) realizadas posteriormente mostraron la oxidación progresiva de la superficie metálica, conduciendo predominantemente a la formación de goethita $(\alpha-\mathrm{FeO} . \mathrm{OH}$, $40 \%$ en masa).

Se estudió en función del tiempo la capacidad del fieltro grafítico recién recubierto catódicamente con hierro en contribuir como contra-electrodo (ánodo) en la generación electrolítica de $\mathrm{H}_{2} \mathrm{O}_{2}$. En esta celda, como electrodo de trabajo responsable de la generación catódica de $\mathrm{H}_{2} \mathrm{O}_{2}$ se usó fieltro grafítico no modificado en contacto con $\mathrm{Na}_{2} \mathrm{SO}_{4}$ acuoso acidificado a $\mathrm{pH}$ 3. Las pruebas se realizaron a diferentes densidades de corriente $(5,10,15$, y $20 \mathrm{~mA} / \mathrm{cm}^{2}$ ). Bajo estas condiciones, el contenido de $\mathrm{H}_{2} \mathrm{O}_{2}$ logró aumentar con la densidad de corriente aplicada, pero decae luego de 120 minutos.

Como modelo en la aplicación del fieltro modificado en el tratamiento electrolítico de aceites y grasas en efluentes acuosos, se preparó una mezcla acuosa de ácido palmítico en forma de una emulsión estabilizada con Tritón X-100. Esta muestra sintética fue tratada electrolíticamente en una celda similar a la usada para la generación de $\mathrm{H}_{2} \mathrm{O}_{2}$. Mediante este tratamiento electrolítico se redujo significativamente los niveles de demanda química de oxígeno (DQO) y de turbidez nefelométrica (NTU) en tiempos relativamente cortos: casi $75 \%$ de reducción para DQO y $90 \%$ de NTU en unos 30 minutos. Tales resultados se 
justifican por una acción combinada del proceso electro-Fenton iniciado en el cátodo de fieltro grafítico, y de la electro-coagulación inducida por la oxidación y disolución de hierro desde el fieltro grafítico modificado con este metal.

\section{AGRADECIMIENTOS}

El presente estudio fue posible gracias al apoyo dado por VRI-PUCP mediante el financiamiento del proyecto DGI 2014-0026, así como al CONCYTEC mediante el convenio 231-2015 FONDECYT.

\section{REFERENCIAS BIBLIOGRÁFICAS}

1. Anglada A, Urtiaga A, Ortiz I. Contributions of electrochemical oxidation to wastewater treatment: fundamentals and review of applications. J Chem Technol Biotechnol. 2009; 84(12): 1747-1753.

2. Zhang G, Yang F, Gao M, Liu L. Electrocatalytic Behavior of the Bare and the Anthraquinonedisulfonate/Polypyrrole Composite Film Modified Graphite Cathodes in the Electro-Fenton System. J Phys Chem C. 2008; 112(24): 8957-8962.

3. Cañizares P, García-Gómez J, Lobato L, Rodrigo M. Electrochemical Oxidation of Aqueous Carboxylic Acid Wastes Using Diamond Thin-Film Electrodes. Ind Eng Chem Res. 2003; 42(5): 956 -962.

4. Yi F, Chen S. Electrochemical treatment of alizarin red S dye wastewater using an activated carbon fiber as anode material. J Porous Mater. 2008; 15(5): 565-569.

5. Mert B, Yonar T, Kiliç M, Kestioğlu K. Pre-treatment studies on olive oil mill effluent using physicochemical, Fenton and Fenton-like oxidations processes. J Hazard Mater. 2010; 174(1-3): 122-128.

6. Panizza M, Cerisola G, Removal of organic pollutants from industrial wastewater by electrogenerated Fenton's reagent. Water Res. 2001; 35(16): 3987-3992

7. Sklari S, Plakas K, Petsi P, Zaspalis V, Karabelas A. Toward the Development of a Novel Electro-Fenton System for Eliminating Toxic Organic Substances from Water. Part 2. Preparation, Characterization, and Evaluation of Iron-Impregnated Carbon Felts as Cathodic Electrodes. Ind Eng Chem Res. 2015; 54(7): 2059-2073.

8. Valto P, Knuutinen J, Alén R, Rantalankila M, Lehmonen J, Grönroos A, et al. Analysis of resin and fatty acids enriched in papermaking process waters. Bio Resources. 2010; 5(1): 172-186.

9. Rivas J, Prazeres A, Carvalho F, Beltrán F. Treatment of Cheese Whey Wastewater: Combined Coagulation-Flocculation and Aerobic Biodegradation. J Agric Food Chem. 2010; 58: 7871-7877.

10. Rivas J, Prazeres A, Carvalho F. Aerobic Biodegradation of Precoagulated Cheese Whey Wastewater. J Agric Food Chem. 2011; 59: 2511-2517.

11. Castro Angulo AG. Degradación de ácido palmítico en medio acuoso mediante el uso de fieltros de grafito modificados. [tesis para optar el título de Magíster] Lima: Pontificia Universidad Católica del Perú (PUCP); 2018. 